\title{
含丙烯酰胺结构的喹唑啉衍生物的合成及抗肿瘤活性研究
}

\author{
张路野 $a, b$
刘丽敏 \\ 张 洋 $a, b$ \\ 汪正捷 $a, b$ \\ 王 涛 $a, b$ \\ 李二冬 $a, b$ \\ 刘秀娟 $a, b$ \\ 郑甲信 $a, b$ \\ 可 钰 $a, b$ \\ 单丽红*,a,d

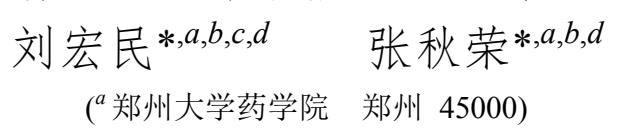 \\ ( ${ }^{b}$ 新药创制与药物安全性评价河南省协同创新中心 郑州 450001) \\ ( ${ }^{c}$ 省部共建食管癌防治国家重点实验室 郑州 450052)

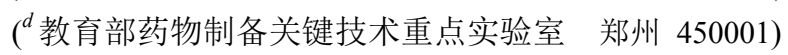

\begin{abstract}
摘要 为了寻找高效低毒的抗肿瘤药物, 设计并合成了一系列新型的含 $N$-(3-丙烯酰胺苯基)乙酰胺结构的哇唑啉类衍 生物, 并采用噻唑蓝(MTT)法测定了目标化合物对 H1975(人肺腺癌细胞系), PC-3(人前列腺癌细胞系), MGC-803(人胃 癌细胞系)三种肿瘤细胞的抗增殖活性. 结果显示大部分化合物具有较好的抗肿瘤活性, 其中 $N-(3-(2-(((4-)(4-$ 氯苯基)氨 基)-7-甲氧基喹唑啉-6-基)氧基)乙酰氨基)苯基)丙烯酰胺(13j)对 H1975, MGC-803 两种细胞显示出最好的抗增殖活性, $\mathrm{IC}_{50}$ 值分别为 $(6.77 \pm 0.65)$ 和 $(4.06 \pm 0.34) \mu \mathrm{mol} / \mathrm{L}$, 其活性均优于阳性对照品吉非替尼, 为抗肿瘤药物的研究提供了线 索.
\end{abstract}

关键词＼cjkstart丙烯酰胺; 喹唑啉; 合成; 抗肿瘤活性

\section{Synthesis and Antitumor Activity of Novel Quinazoline Derivatives Containing Acrylamide}

\author{
Zhang, Luye ${ }^{a, b}$ \\ Zhang, Yang ${ }^{a, b}$ \\ Wang, Zhengjie ${ }^{a, b}$ \\ Wang, Tao ${ }^{a, b}$ \\ Li, Erdong ${ }^{a, b}$ \\ Liu, $\operatorname{Limin}^{a, b}$ \\ Liu, Xiujuan ${ }^{a, b}$ \\ Zheng, Jiaxin ${ }^{a, b}$ \\ $\mathrm{Ke}, \mathrm{Yu}^{a, b}$ \\ Shan, Lihong ${ }^{*, a, d}$ \\ Liu, Hongmin $*, a, b, c, d$ \\ Zhang, Qiurong ${ }^{*, a, b, d}$ \\ ( ${ }^{a}$ School of Pharmaceutical Sciences, Zhengzhou University, Zhengzhou 450001) \\ ( ${ }^{b}$ Collaborative Innovation Center of New Drug Research and Safety Evaluation of Henan Province, Zhengzhou 450001) \\ ( ${ }^{c}$ State Key Laboratory of Esophageal Cancer Prevention \& Treatment, Zhengzhou 450052) \\ ( ${ }^{d}$ Key Laboratory of Advanced Drug Preparation Technologies, Ministry of Education, Zhengzhou 450001)
}

\begin{abstract}
In order to find efficient and low toxicity anti-tumor drugs, a series of novel quinazoline derivatives containing $N$-(3-aminophenyl)acrylamide were synthesized and their antiproliferative activities were evaluated against three human cancer cell lines (H1975, PC-3, MGC-803) by using methyl thiazolyl tetrazolium (MTT) assay. The results showed that most compounds exhibited better antiproliferative activities against the four human tumor cell lines. Among them, $N$-(3-(2-((4-((4-chlorophenyl)amino)-7-methoxy- quinazolin-6-yl)oxy)acetamido)phenyl)acrylamide (13j) showed the best antiproliferative activity against H1975 and MGC-803 cancer cell lines with $\mathrm{IC}_{50}$ values of $(6.77 \pm 0.65)$ and (4.06 \pm 0.34$)$ $\mu \mathrm{mol} / \mathrm{L}$, respectively. Its activity was better than the positive control gefitinib. In a nutshell, this work provides clues to discover antitumor agent based on the quinazoline scaffold.
\end{abstract}

Keywords acrylamide; quinazoline; synthesis; antiproliferative activity

\footnotetext{
* Corresponding authors. E-mail: zqr409@yeah.net; shlh@zzu.edu.cn; liuhm@zzu.edu.cn

Received May 28, 2020; revised June 17, 2020; published online June 30, 2020.

Project supported by the National Natural Science Foundation of China (No. U1904163), and the Opening Fund from State Key Laboratory of Esophageal Cancer Prevention \& Treatment (No. K2020000X).

国家自然科学基金(No. U1904163)、省部共建食管癌防治国家重点实验室开放基金(No. K2020000X)资助项目.
} 


\section{Introduction}

Currently, the cancer is the second major cause of human death after cardiovascular disease. ${ }^{[1]}$ Statistics show that approximately 13.15 million people will die from cancer until 2035. ${ }^{[2]}$ Chemotherapy is a very important method in clinic. However, Many anti-tumor drugs are prone to drug resistance. ${ }^{[3]}$ Therefore, finding efficient and low toxicity anti-tumor drugs is of great significance for the treatment of tumors.

Quinazoline derivatives are important nitrogen-containing heterocycles ${ }^{[4]}$ with a variety of pharmacological properties, such as antimalarial, ${ }^{[5-6]}$ antibacterial, ${ }^{[7-8]}$ anti-inflammatory, ${ }^{[9-10]}$ anticonvulsant, ${ }^{[11-12]}$ antihypertensive, ${ }^{[13]}$ anti-diabetic, ${ }^{[14]}$ cholinesterase inhibition ${ }^{[15-16]}$ and antitumor. ${ }^{[17-18]}$ With the remarkable progress made in recent years, researchers have found that 4-aminoquinazoline plays an important role in inhibiting epidermal growth factor receptor tyrosine kinase. Some of the drugs with 4-aminoquinazolineare are effective for the treatment of non-small cell lung cancers (NSCLCs), such as erlotinib, gefitinib, lapatinib, and afatinib (Figure 1). ${ }^{[19]}$

Acrylamide substituted derivatives play an important role in pharmaceutical chemistry. For example, the third generation epidermal growth factor receptor (EGFR) inhibitors all have the acrylamide moiety (Figure 1). ${ }^{[20]}$

Therefore, a series of quinazoline derivatives containing $\mathrm{N}$-(3-aminophenyl)acrylamide were synthesized by using the combination principles and the antiproliferative activity of target compounds in vitro was evaluated by methyl thiazolyl tetrazolium (MTT) assay .

\section{Results and discussion}

\subsection{Chemistry}

The synthetic strategy to prepare the target compounds is depicted in Scheme 1. Firstly, 6-hydroxy-7-methoxyquinazolin-4(3H)-one and pyridine were dissolved in acetic anhydride at $80{ }^{\circ} \mathrm{C}$ for $4 \mathrm{~h}$ to obtained compound 9 . Next, phosphorus oxychloride was added to compound $\mathbf{9}$ and the temperature was slowly raised to $80{ }^{\circ} \mathrm{C}$ and kept the reaction for $2 \mathrm{~h}$ to obtained compound 10. Then compounds 11a $\sim 11 \mathrm{v}$ were acquired from the reaction of compound 10 with substituted aniline in isopropanol at $80{ }^{\circ} \mathrm{C}$ for $1 \mathrm{~h}$. $\mathrm{NH}_{3} \cdot \mathrm{H}_{2} \mathrm{O}$ was added to compounds $\mathbf{1 1 a} \sim \mathbf{1 1 q}$ in $\mathrm{CH}_{3} \mathrm{OH}$ at $75{ }^{\circ} \mathrm{C}$ for $2 \mathrm{~h}$ to obtained compounds $12 \mathrm{a} \sim \mathbf{1 2 q}$. Finally, compounds $12 \mathbf{a} \sim 12 \mathbf{q}$ and commercially available $N$-(3-(2chloroacetamido)phenyl)acrylamide were added to $N, N^{\prime}$-dimethylformamide (DMF) and the temperature was raised to $90{ }^{\circ} \mathrm{C}$ for $2 \mathrm{~h}$ to get the target compounds $13 \mathbf{a} \sim 13 \mathbf{~}$. The structures of target compounds were confirmed by ${ }^{1} \mathrm{H}$ NMR, ${ }^{13} \mathrm{C}$ NMR and HRMS.

\subsection{Anti-tumor activity}

In order to explore the antiproliferative activity of the target compounds, compounds $13 \mathbf{1 3} \sim \mathbf{1 3 q}$ were evaluated against three human cancer cell lines including H1975 (human lung cancer cell line), PC-3 (human prostate cancer cell line), MGC-803 (human gastric carcinoma cell line) by using MTT assay. Gefitinib was employed as the positive control. The results are shown in Table 1.

In order to explore the structure-activity relationship, different substituents were introduced to quinazoline scaf-<smiles>C#Cc1cccc(Nc2ncnc3cc(OCCOC)c(OCCOC)cc23)c1</smiles><smiles>CN(C)C/C=C/C(=O)Nc1cc2c(Nc3ccc(F)c(Cl)c3)ncnc2cc1OC1CCCC1</smiles><smiles>CCCCC/C=C\C(=O)Nc1cc(Nc2nccc(-c3cn(C)c4ccccc34)n2)c(OC)cc1N(C)CCN(C)C</smiles>

4 Afatinib

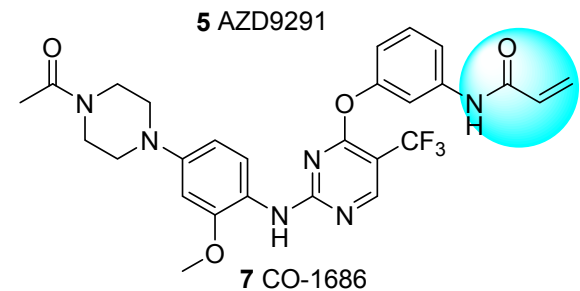<smiles>CCCCCCC=CC(=O)Nc1cccc(Oc2nc(Nc3ccc(N4CCN(C)CC4)cc3OC)ncc2Cl)c1</smiles>

Figure 1 Structures of some 4-aminoquinazoline derivatives containing benzothiazole 
<smiles>COc1cc2nc[nH]c(=O)c2cc1OCC(C)(C)CCOc1cc2nc[nH]c(=O)c2cc1OC(C)=O</smiles><smiles>[R]c1ccc(Nc2ncnc3cc(OC)c(O)cc23)cc1O</smiles><smiles>[R]c1ccc(Nc2ncnc3cc(OC)c(OCC(=O)Nc4cccc(NC(=O)C=C)c4)cc23)cc1</smiles>

$13 a \sim 13 q$

Reagents and conditions: (i) $\left(\mathrm{CH}_{3} \mathrm{CO}\right)_{2} \mathrm{O}$, pyridine, $80{ }^{\circ} \mathrm{C}, 4 \mathrm{~h}$; (ii) $\mathrm{POCl}_{3}, 80{ }^{\circ} \mathrm{C}, 2 \mathrm{~h}$; (iii) substituted aniline, isopropanol, $80{ }^{\circ} \mathrm{C}, 1 \mathrm{~h}$; (iv) $\mathrm{NH}_{3}{ }^{\circ} \mathrm{H}_{2} \mathrm{O}, \mathrm{CH}_{3} \mathrm{OH}, 75{ }^{\circ} \mathrm{C}, 2 \mathrm{~h}$; (V) $\mathrm{N}$-(3-(2-chloroacetamido)phenyl)acrylamide, DMF, $90{ }^{\circ} \mathrm{C}, 2 \mathrm{~h}$

Scheme 1 Synthesis of compounds 13a $\sim \mathbf{1 3 q}$

fold. As shown in Table 1, the majority of the compounds exhibited moderate antiproliferative activity against three human cancer cell lines. Among all the target compounds, compound $\mathbf{1 3} \mathbf{j}$ showed the best cytotoxicity against the tested cell lines (H1975, MGC-803) with $\mathrm{IC}_{50}$ values of $(6.77 \pm 0.65)$ and $(4.06 \pm 0.34) \mu \mathrm{mol} / \mathrm{L}$, which was better than gefitinib.

From the biological data of compounds 13a, 13b, 13c, it is known that the contribution to enhance antitumor ac- tivity was $\mathrm{F}<\mathrm{Cl}<\mathrm{Br}$, when the halogen atoms at 2-position of benzene. From the biological data of compound 13g, it is concluded that compounds with nitro at $\mathrm{R}$ of benzene exhibited low cytotoxicity. From the biological data of compounds $\mathbf{1 3 h}$ and $\mathbf{1 3 m}$, the results revealed that the methoxy at 3-position of benzene had better cytotoxic activity for cancer cells than that at 4-position. From the biological data of compounds $13 \mathrm{I}$ and $\mathbf{1 3} \mathrm{m}$, the results revealed that the methyl at 4-position of benzene had better cytotoxic activity against the tested cell lines (PC-3, MGC-803) than the methoxy at 4-position.

\section{Conclusion}

In conclusion, a series of novel quinazoline derivatives containing acrylamide were synthesized and evaluated for their cytotoxic activity against H1975, PC-3 and MGC-803 cancer cells using MTT assay.

Among all the tested compounds, compound 13j showed the most potent anti-proliferative activity against the tested cells. This work provided clues to discover antitumor agent based on the quinazoline scaffold.

\section{Experimental section}

\subsection{Instruments and reagents}

Melting points were determined on an X-5 micro-melting apparatus and are uncorrected. ${ }^{1} \mathrm{H}$ NMR and ${ }^{13} \mathrm{C}$ NMR spectra were recorded on a Bruker $400 \mathrm{MHz}$ and $101 \mathrm{MHz}$ spectrometer, respectively. High resolution mass spectra (HRMS) were recorded on a Waters Micro-mass Q-T of Micro-mass spectrometer by electrospray ionization (ESI). Reagents and solvents were purchased from commercial sources and were used without further purification. Column

Table 1 Antiproliferative activity of target compounds $\mathbf{1 3 a} \sim \mathbf{1 3 q}$ against three cancer cell lines

\begin{tabular}{|c|c|c|c|c|}
\hline \multirow{2}{*}{ Compound } & \multirow{2}{*}{$\mathrm{R}$} & \multicolumn{3}{|c|}{$\mathrm{IC}_{50}{ }^{a} /\left(\mu \mathrm{mol} \cdot \mathrm{L}^{-1}\right)$} \\
\hline & & H1975 & PC-3 & MGC-803 \\
\hline $13 a$ & $2-\mathrm{F}$ & $>50$ & $23.11 \pm 0.54$ & $32.54 \pm 0.92$ \\
\hline $13 b$ & $2-\mathrm{Cl}$ & $>50$ & $9.79 \pm 0.61$ & $30.73 \pm 1.21$ \\
\hline $13 \mathrm{c}$ & $2-\mathrm{Br}$ & $>50$ & $8.42 \pm 0.70$ & $16.85 \pm 0.54$ \\
\hline 13d & $3-\mathrm{F}$ & $29.75 \pm 0.56$ & $28.24 \pm 0.86$ & $18.63 \pm 1.18$ \\
\hline $13 \mathrm{e}$ & $3-\mathrm{Cl}$ & $>50$ & $>50$ & $24.28 \pm 0.89$ \\
\hline $13 f$ & $3-\mathrm{Br}$ & $>50$ & $30.81 \pm 0.58$ & $>50$ \\
\hline $13 g$ & $3-\mathrm{NO}_{2}$ & $>50$ & $>50$ & $49.03 \pm 0.72$ \\
\hline $13 \mathrm{~h}$ & $3-\mathrm{OCH}_{3}$ & $8.94 \pm 1.02$ & $14.53 \pm 0.64$ & $10.84 \pm 0.71$ \\
\hline $13 \mathbf{i}$ & $4-\mathrm{F}$ & $>50$ & $>50$ & $20.79 \pm 1.19$ \\
\hline $13 \mathbf{j}$ & $4-\mathrm{Cl}$ & $6.77 \pm 0.65$ & $9.89 \pm 0.75$ & $4.06 \pm 0.34$ \\
\hline $13 k$ & $4-\mathrm{Br}$ & $15.89 \pm 1.24$ & $23.28 \pm 0.87$ & $42.00 \pm 1.03$ \\
\hline $13 I$ & $4-\mathrm{CH}_{3}$ & $16.41 \pm 1.14$ & $13.4 \pm 0.98$ & $9.98 \pm 1.36$ \\
\hline $13 m$ & $4-\mathrm{OCH}_{3}$ & $13.2 \pm 0.79$ & $41.92 \pm 0.74$ & $40.14 \pm 1.45$ \\
\hline $13 n$ & $2,4-\mathrm{Cl}_{2}$ & $>50$ & $28.42 \pm 0.97$ & $49.61 \pm 0.78$ \\
\hline 130 & $3,4-\mathrm{Cl}_{2}$ & $>50$ & $29.69 \pm 1.04$ & $14.81 \pm 1.15$ \\
\hline $13 p$ & $3-\mathrm{Cl}-4-\mathrm{F}$ & $>50$ & $34.80 \pm 0.53$ & $12.84 \pm 1.08$ \\
\hline $13 q$ & $3,4,5-\left(\mathrm{OCH}_{3}\right)_{3}$ & $>50$ & $33.62 \pm 0.81$ & $41.30 \pm 0.55$ \\
\hline Gefitinib $^{b}$ & - & $9.20 \pm 0.76$ & $8.92 \pm 0.41$ & $8.19 \pm 0.67$ \\
\hline
\end{tabular}

${ }^{a}$ Antiproliferative was assayed by exposure for $72 \mathrm{~h}$ to substances and expressed as concentration required to inhibit tumor cells proliferation by $50 \%\left(\mathrm{IC}_{50}\right) .{ }^{b}$ Used as a positive control. 
chromatography was carried out on $200 \sim 300$ mesh silica gel (Qingdao Haiyang Chemical, China). Reactions were monitored by thin-layer chromatography (TLC) on 0.25 mm silicagel plates (GF254) and visualized under UV light.

7-Methoxy-4-oxo-3,4-dihydroquinazolin-6-ylacetate (9), 4-chloro-7-methoxyquinazolin-6-yl acetate (10), compounds 11a, 11d, 11e, 11f, 11g, 11i, 11l, 11m, 11o, $11 \mathrm{p}, 11 \mathrm{q}$, and compounds $12 \mathrm{a}, 12 \mathrm{~d}, 12 \mathrm{e}, 12 \mathrm{f}, 12 \mathrm{~g}, 12 \mathrm{i}$, 12l, 12m, 12o, 12p, 12q were synthesized according to the published literature. ${ }^{[21]}$ Compounds 11b, 11c, 11j, $11 \mathrm{k}$, and compounds $12 \mathrm{~b}, 12 \mathrm{c}, 12 \mathbf{j}, 12 \mathrm{k}$ were synthesized according to the published literature. ${ }^{[22]}$ Compounds 11n, 11h, and compounds 12n, 12h were synthesized according to the published literature. ${ }^{[23]}$ The characterization data of all these compounds were consistent with the literature.

3.2 General procedure for synthesis of target compounds $13 a \sim 13 q$

$N$-(3-(2-Chloroacetamido)phenyl)acrylamide $\quad(0.35 \mathrm{mmol})$ was dissolved in $4 \mathrm{~mL}$ of $N, N$-dimethylformamide at room temperature. Then, compounds $\mathbf{1 2 a} \sim \mathbf{1 2 q}(0.39 \mathrm{mmol})$ were added dropwise to the above system. The reaction was carried out at $90{ }^{\circ} \mathrm{C}$ for $2 \mathrm{~h}$. After the reaction was completed (TLC detection reaction), it was cooled to room temperature. An appropriate amount of water was added into the system and white solids were obtained. The precipitate was collected by filtration. Next, crude compound was subjected to column chromatography (petroleum ether/ethyl acetate, $V: V=3: 1$ ). Concentrated eluent to give solid compounds $\mathbf{1 3 a} \sim \mathbf{1 3 q}$.

$N$-(3-(2-((4-((2-Fluorophenyl)amino)-7-methoxyquinazolin-6-yl)oxy)acetamido)phenyl)acrylamide (13a): White solid, yield 78.2\%. m.p. 258 259 ${ }^{\circ} \mathrm{C} ;{ }^{1} \mathrm{H}$ NMR (400 MHz, DMSO- $\left.d_{6}\right) \delta: 10.19(\mathrm{~s}, 1 \mathrm{H}), 10.14(\mathrm{~s}, 1 \mathrm{H}), 9.50(\mathrm{~s}, 1 \mathrm{H}), 8.38$ (s, 1H), 8.10 (s, 1H), 7.94 (s, 1H), 7.55 (t, $J=7.8 \mathrm{~Hz}, 1 \mathrm{H})$, $7.43(\mathrm{~d}, J=8.1 \mathrm{~Hz}, 1 \mathrm{H}), 7.37$ (d, $J=8.2 \mathrm{~Hz}, 1 \mathrm{H}), 7.31$ (dt, $J=11.9,4.8 \mathrm{~Hz}, 3 \mathrm{H}), 7.26(\mathrm{~s}, 2 \mathrm{H}), 6.46(\mathrm{dd}, J=17.0,10.1$ $\mathrm{Hz}, 1 \mathrm{H}), 6.27$ (dd, $J=17.0,2.1 \mathrm{~Hz}, 1 \mathrm{H}), 5.75$ (dd, $J=10.0$, $2.1 \mathrm{~Hz}, 1 \mathrm{H}), 4.90(\mathrm{~s}, 2 \mathrm{H}), 4.00(\mathrm{~s}, 3 \mathrm{H}) ;{ }^{13} \mathrm{C} \mathrm{NMR}(101 \mathrm{MHz}$, DMSO- $\left.d_{6}\right) \delta: 165.9(\mathrm{~d}, J=276.7 \mathrm{~Hz}), 158.1,157.3,155.6$, $154.4,153.4,147.5,147.2,139.6,138.7,131.8,129.0$, 128.4, 127.1, 126.9, 126.5, 124.4 (d, $J=3.0 \mathrm{~Hz}), 116.0$ (d, $J=20.2$. Hz), 114.7, 110.6, 108.4, 107.4, 103.9, 68.2, 55.9. HRMS (ESI) calcd for $\mathrm{C}_{26} \mathrm{H}_{23} \mathrm{FN}_{5} \mathrm{O}_{4}[\mathrm{M}+\mathrm{H}]^{+}: 488.1734$, found 488.1733 .

$\mathrm{N}$-(3-(2-((4-((2-Chlorophenyl)amino)-7-methoxyquinazolin-6-yl)oxy)acetamido)phenyl)acrylamide (13b): White solid, yield 77.1\%. m.p. 236 237 ${ }^{\circ} \mathrm{C} ;{ }^{1} \mathrm{H}$ NMR $(400 \mathrm{MHz}$, DMSO- $\left.d_{6}\right) \delta: 10.18(\mathrm{~s}, 1 \mathrm{H}), 10.13(\mathrm{~s}, 1 \mathrm{H}), 9.50(\mathrm{~s}, 1 \mathrm{H}), 8.34$ $(\mathrm{s}, 1 \mathrm{H}), 8.09(\mathrm{~s}, 1 \mathrm{H}), 7.94(\mathrm{~s}, 1 \mathrm{H}), 7.60 \sim 7.55(\mathrm{~m}, 2 \mathrm{H}), 7.42$ $(\mathrm{d}, J=8.1 \mathrm{~Hz}, 2 \mathrm{H}), 7.36(\mathrm{~d}, J=8.3 \mathrm{~Hz}, 1 \mathrm{H}), 7.33$ (d, $J=7.7$ $\mathrm{Hz}, 1 \mathrm{H}), 7.28$ (d, $J=8.5 \mathrm{~Hz}, 1 \mathrm{H}), 7.26(\mathrm{~s}, 1 \mathrm{H}), 6.45$ (dd, $J=17.0,10.1 \mathrm{~Hz}, 1 \mathrm{H}), 6.26(\mathrm{dd}, J=17.0,2.2 \mathrm{~Hz}, 1 \mathrm{H}), 5.75$ $(\mathrm{dd}, J=10.1,2.2 \mathrm{~Hz}, 1 \mathrm{H}), 4.89(\mathrm{~s}, 2 \mathrm{H}), 3.99(\mathrm{~s}, 3 \mathrm{H}) ;{ }^{13} \mathrm{C}$ NMR (101 MHz, DMSO- $\left.d_{6}\right) \delta$ : 165.8, 163.1, 157.5, 154.4, $153.4,147.5,147.2,139.4,138.7,136.1,131.8,130.8$, $129.7,129.0,127.6,127.5,126.8,114.7,114.7,110.6$,
108.3, 107.4, 103.9, 68.2, 55.9. HRMS (ESI) calcd for $\mathrm{C}_{26} \mathrm{H}_{23} \mathrm{ClN}_{5} \mathrm{O}_{4}[\mathrm{M}+\mathrm{H}]^{+}$504.1439, found 504.1440.

$\mathrm{N}$-(3-(2-((4-((2-Bromophenyl)amino)-7-methoxyquinazolin-6-yl)oxy)acetamido)phenyl)acrylamide (13c): White solid, yield 73.8\%. m.p. 236 $237{ }^{\circ} \mathrm{C} ;{ }^{1} \mathrm{H}$ NMR (400 MHz, DMSO- $\left.d_{6}\right) \delta: 10.18(\mathrm{~s}, 1 \mathrm{H}), 10.13(\mathrm{~s}, 1 \mathrm{H}), 9.48(\mathrm{~s}, 1 \mathrm{H}), 8.33$ (s, 1H), 8.09 (s, 1H), $7.93(\mathrm{~s}, 1 \mathrm{H}), 7.73(\mathrm{~d}, J=8.0 \mathrm{~Hz}, 1 \mathrm{H})$, 7.57 (d, $J=7.9 \mathrm{~Hz}, 1 \mathrm{H}), 7.44$ (dd, $J=15.4,7.8 \mathrm{~Hz}, 2 \mathrm{H})$, 7.36 (d, $J=8.3 \mathrm{~Hz}, 1 \mathrm{H}), 7.26$ (d, $J=7.7 \mathrm{~Hz}, 3 \mathrm{H}), 6.45$ (dd, $J=17.0,10.1 \mathrm{~Hz}, 1 \mathrm{H}), 6.30 \sim 6.21(\mathrm{~m}, 1 \mathrm{H}), 5.75(\mathrm{dd}, J=$ 10.1, 2.2 Hz, 1H), 4.89 (s, 2H), 3.99 (s, 3H); ${ }^{13} \mathrm{C} \mathrm{NMR} \mathrm{(101}$ MHz, DMSO- $\left.d_{6}\right) \delta: 165.8,163.1,157.5,154.4,153.4$, $147.4,139.4,138.7,137.5,132.8,131.8,123.0,129.0$, $128.2,127.9,126.8,121.9,114.7,114.7,110.6,108.3$, 107.4, 103.8, 68.1, 55.9. HRMS (ESI) calcd for $\mathrm{C}_{26} \mathrm{H}_{23} \mathrm{Br}-$ $\mathrm{N}_{5} \mathrm{O}_{4}[\mathrm{M}+\mathrm{H}]^{+}$548.0933, found 548.0932.

$\mathrm{N}$-(3-(2-((4-((3-Fluorophenyl)amino)-7-methoxyquinazolin-6-yl)oxy)acetamido)phenyl)acrylamide (13d): White solid, yield 76.8\% m.p. 183 $184{ }^{\circ} \mathrm{C}$; ${ }^{1} \mathrm{H}$ NMR (400 MHz, DMSO- $\left.d_{6}\right) \delta: 10.19(\mathrm{~s}, 1 \mathrm{H}), 10.13(\mathrm{~s}, 1 \mathrm{H}), 9.58(\mathrm{~s}, 1 \mathrm{H}), 8.56$ $(\mathrm{s}, 1 \mathrm{H}), 8.12(\mathrm{~s}, 1 \mathrm{H}), 7.98(\mathrm{~s}, 1 \mathrm{H}), 7.90(\mathrm{dt}, J=11.9,2.4 \mathrm{~Hz}$, $1 \mathrm{H}), 7.60$ (d, $J=8.3 \mathrm{~Hz}, 1 \mathrm{H}), 7.43$ (dt, $J=7.2,3.4 \mathrm{~Hz}, 2 \mathrm{H})$, 7.38 (d, $J=7.7 \mathrm{~Hz}, 1 \mathrm{H}), 7.29$ (t, $J=4.1 \mathrm{~Hz}, 2 \mathrm{H}), 6.93$ (td, $J=8.5,2.4 \mathrm{~Hz}, 1 \mathrm{H}), 6.46(\mathrm{dd}, J=17.0,10.1 \mathrm{~Hz}, 1 \mathrm{H}), 6.27$ (dd, $J=17.0,2.2 \mathrm{~Hz}, 1 \mathrm{H}), 5.76(\mathrm{dd}, J=10.1,2.2 \mathrm{~Hz}, 1 \mathrm{H})$, $4.91(\mathrm{~s}, 2 \mathrm{H}), 4.01(\mathrm{~s}, 3 \mathrm{H}) ;{ }^{13} \mathrm{C}$ NMR (101 MHz, DMSO- $\left.d_{6}\right)$ $165.8,163.1$ (d, $J=237.3 \mathrm{~Hz}), 156.2,154.5,153.0,147.5$, $147.4,141.2,139.4,138.6,131.8,129.9$ (d, $J=10.1 \mathrm{~Hz})$, $129.0,126.8,117.5,114.7,110.6,109.6,109.4,108.8$, 108.5, 107.6, 104.2, 68.6, 56.0. HRMS (ESI) calcd for $\mathrm{C}_{26} \mathrm{H}_{23} \mathrm{FN}_{5} \mathrm{O}_{4}[\mathrm{M}+\mathrm{H}]^{+}$488.1734, found 488.1735.

$\mathrm{N}$-(3-(2-((4-((3-Chlorophenyl)amino)-7-methoxyquinazolin-6-yl)oxy)acetamido)phenyl)acrylamide (13e): White solid, yield 72.9\%. m.p. 270 $271{ }^{\circ} \mathrm{C} ;{ }^{1} \mathrm{H} \mathrm{NMR}(400 \mathrm{MHz}$, DMSO- $\left.d_{6}\right) \delta: 10.19(\mathrm{~s}, 1 \mathrm{H}), 10.13(\mathrm{~s}, 1 \mathrm{H}), 9.55(\mathrm{~s}, 1 \mathrm{H}), 8.56$ $(\mathrm{s}, 1 \mathrm{H}), 8.11(\mathrm{~s}, 1 \mathrm{H}), 8.04(\mathrm{t}, J=2.0 \mathrm{~Hz}, 1 \mathrm{H}), 7.97(\mathrm{~s}, 1 \mathrm{H})$, 7.79 (d, $J=8.3 \mathrm{~Hz}, 1 \mathrm{H}), 7.43$ (d, $J=8.2 \mathrm{~Hz}, 2 \mathrm{H}), 7.38$ (d, $J=9.5 \mathrm{~Hz}, 1 \mathrm{H}), 7.29$ (s, 2H), $7.18 \sim 7.13(\mathrm{~m}, 1 \mathrm{H}), 6.46(\mathrm{dd}$, $J=17.0,10.1 \mathrm{~Hz}, 1 \mathrm{H}), 6.27(\mathrm{dd}, J=17.0,2.1 \mathrm{~Hz}, 1 \mathrm{H}), 5.76$ $(\mathrm{dd}, J=10.1,2.1 \mathrm{~Hz}, 1 \mathrm{H}), 4.91(\mathrm{~s}, 2 \mathrm{H}), 4.01(\mathrm{~s}, 3 \mathrm{H}) ;{ }^{13} \mathrm{C}$ NMR (101 MHz, DMSO- $\left.d_{6}\right) \delta: 165.8,163.1,156.1,154.5$, $153.0,147.5,147.4,141.0,139.4,138.6,132.7131 .8$, $130.0,129.0,126.9,122.8,121.2,120.2,114.8,110.6$, 108.7, 107.6, 104.1, 68.6, 56.0. HRMS (ESI) calcd for $\mathrm{C}_{26} \mathrm{H}_{23} \mathrm{ClN}_{5} \mathrm{O}_{4}[\mathrm{M}+\mathrm{H}]^{+}$504.1439, found 504.1438.

$\mathrm{N}$-(3-(2-((4-((3-Bromophenyl)amino)-7-methoxyquinazolin-6-yl)oxy)acetamido)phenyl)acrylamide (13f): White solid, yield 71.5\%. m.p. $246 \sim 247{ }^{\circ} \mathrm{C} ;{ }^{1} \mathrm{H}$ NMR $(400$ MHz, DMSO-d $) \delta: 10.20(\mathrm{~s}, 1 \mathrm{H}), 10.12(\mathrm{~s}, 1 \mathrm{H}), 9.54(\mathrm{~s}$, $1 \mathrm{H}), 8.56(\mathrm{~d}, J=2.6 \mathrm{~Hz}, 1 \mathrm{H}), 8.18 \sim 8.10(\mathrm{~m}, 2 \mathrm{H}), 7.96(\mathrm{~s}$, $1 \mathrm{H}), 7.88 \sim 7.83(\mathrm{~m}, 1 \mathrm{H}), 7.44(\mathrm{~d}, J=8.1 \mathrm{~Hz}, 1 \mathrm{H}), 7.39$ (d, $J=8.5 \mathrm{~Hz}, 1 \mathrm{H}), 7.35$ (d, $J=7.8 \mathrm{~Hz}, 1 \mathrm{H}), 7.30$ (d, $J=8.5$ $\mathrm{Hz}, 3 \mathrm{H}), 6.47$ (dd, $J=17.0,10.0 \mathrm{~Hz}, 1 \mathrm{H}), 6.28(\mathrm{dd}, J=17.0$, $2.3 \mathrm{~Hz}, 1 \mathrm{H}), 5.76(\mathrm{dd}, J=10.1,2.2 \mathrm{~Hz}, 1 \mathrm{H}), 4.91(\mathrm{~s}, 2 \mathrm{H})$, $4.01(\mathrm{~s}, 3 \mathrm{H}) ;{ }^{13} \mathrm{C}$ NMR (101 MHz, DMSO- $\left.d_{6}\right) \delta: 165.8$, $163.1,156.1,154.5,153.0,147.5,147.5,141.1,139.4$, $138.6,131.8,130.3,129.0,126.9,125.7,124.0,121.2$, 
120.6, 114.8, 110.6, 108.7, 107.6, 104.1, 68.6, 56.0. HRMS (ESI) calcd for $\mathrm{C}_{26} \mathrm{H}_{23} \mathrm{BrN}_{5} \mathrm{O}_{4}[\mathrm{M}+\mathrm{H}]^{+}$548.0933, found 548.0932.

$\mathrm{N}$-(3-(2-((7-Methoxy-4-((3-nitrophenyl)amino)quinazolin-6-yl)oxy)acetamido)phenyl)acrylamide (13g): White solid, yield 67.6\%. m.p. 247 248 ${ }^{\circ} \mathrm{C} ;{ }^{1} \mathrm{H}$ NMR (400 $\left.\mathrm{MHz}, \mathrm{DMSO}-d_{6}\right) \delta: 10.19(\mathrm{~s}, 1 \mathrm{H}), 10.16(\mathrm{~s}, 1 \mathrm{H}), 9.96$ (s, $1 \mathrm{H}), 8.66$ (s, 1H), 8.29 (d, $J=9.0 \mathrm{~Hz}, 2 \mathrm{H}), 8.19$ (d, $J=9.0$ $\mathrm{Hz}, 2 \mathrm{H}), 8.11(\mathrm{~s}, 1 \mathrm{H}), 8.00(\mathrm{~s}, 1 \mathrm{H}), 7.39$ (t, $J=8.4 \mathrm{~Hz}, 2 \mathrm{H})$, 7.34 (s, 1H), 7.28 (t, $J=8.1 \mathrm{~Hz}, 1 \mathrm{H}), 6.45$ (dd, $J=17.0$, $10.1 \mathrm{~Hz}, 1 \mathrm{H}), 6.26(\mathrm{dd}, J=17.0,2.1 \mathrm{~Hz}, 1 \mathrm{H}), 5.76(\mathrm{dd}, J=$ 10.0, $2.0 \mathrm{~Hz}, 1 \mathrm{H}), 4.93(\mathrm{~s}, 2 \mathrm{H}), 4.02(\mathrm{~s}, 3 \mathrm{H}) ;{ }^{13} \mathrm{C} \mathrm{NMR}$ (101 MHz, DMSO- $\left.d_{6}\right) \delta:{ }^{13} \mathrm{C}$ NMR (101 MHz, DMSO) $\delta$ : $165.7,163.1,155.7,155.2,154.9,152.8,152.7,147.9$, $147.8,146.2,141.5,139.4,138.6,131.8,129.1,126.9$, 124.6, 120.5, 114.8, 114.7, 110.6, 107.6, 104.1, 68.6, 56.1. HRMS (ESI) calcd for $\mathrm{C}_{26} \mathrm{H}_{23} \mathrm{~N}_{6} \mathrm{O}_{6}[\mathrm{M}+\mathrm{H}]^{+}$515.1679, found 515.1677 .

$N$-(3-(2-((7-Methoxy-4-((3-methoxyphenyl)amino)quinazolin-6-yl)oxy)acetamido)phenyl)acrylamide (13h): White solid, yield $72.8 \%$. m.p. $187 \sim 188{ }^{\circ} \mathrm{C} ;{ }^{1} \mathrm{H}$ NMR $\left(400 \mathrm{MHz}, \mathrm{DMSO}-d_{6}\right) \delta: 10.19(\mathrm{~s}, 1 \mathrm{H}), 10.13(\mathrm{~s}, 1 \mathrm{H}), 9.44$ $(\mathrm{s}, 1 \mathrm{H}), 8.50(\mathrm{~d}, J=2.6 \mathrm{~Hz}, 1 \mathrm{H}), 8.11(\mathrm{~s}, 1 \mathrm{H}), 7.99(\mathrm{~s}, 1 \mathrm{H})$, $7.49(\mathrm{~d}, J=2.4 \mathrm{~Hz}, 1 \mathrm{H}), 7.45 \sim 7.35(\mathrm{~m}, 3 \mathrm{H}), 7.32 \sim 7.29$ $(\mathrm{m}, 1 \mathrm{H}), 7.27(\mathrm{~d}, J=6.1 \mathrm{~Hz}, 2 \mathrm{H}), 6.70(\mathrm{dd}, J=8.3,2.4 \mathrm{~Hz}$, $1 \mathrm{H}), 6.46(\mathrm{dd}, J=17.0,10.1 \mathrm{~Hz}, 1 \mathrm{H}), 6.27(\mathrm{dd}, J=17.0$, $2.2 \mathrm{~Hz}, 1 \mathrm{H}), 5.75(\mathrm{dd}, J=9.9,2.2 \mathrm{~Hz}, 1 \mathrm{H}), 4.91(\mathrm{~s}, 2 \mathrm{H})$, $4.00(\mathrm{~s}, 3 \mathrm{H}), 3.78$ (s, 3H); ${ }^{13} \mathrm{C}$ NMR (101 MHz, DMSO- $\left.d_{6}\right)$ $\delta: 165.9,163.1,159.4,156.4,154.4,153.2,147.4,140.5$, $139.4,138.6,131.8,129.1,129.0,126.8,114.8,114.5$, 113.8, 110.6, 108.7, 108.6, 108.1, 107.6, 104.3, 68.6, 56.0, 55.1. HRMS (ESI) calcd for $\mathrm{C}_{27} \mathrm{H}_{26} \mathrm{~N}_{5} \mathrm{O}_{5}[\mathrm{M}+\mathrm{H}]^{+}$ 500.1934 , found 500.1935 .

$N-(3-(2-((4-((4-F l u o r o p h e n y l) a m i n o)-7-m e t h o x y-$ quinazolin-6-yl)oxy)acetamido)phenyl)acrylamide (13i): White solid, yield $75.7 \%$. m.p. $251 \sim 252{ }^{\circ} \mathrm{C}$; ${ }^{1} \mathrm{H}$ NMR $\left(400 \mathrm{MHz}, \mathrm{DMSO}-d_{6}\right) \delta: 10.19(\mathrm{~s}, 1 \mathrm{H}), 10.12(\mathrm{~s}, 1 \mathrm{H}), 9.50$ $(\mathrm{s}, 1 \mathrm{H}), 8.46(\mathrm{~s}, 1 \mathrm{H}), 8.11(\mathrm{~d}, J=2.2 \mathrm{~Hz}, 1 \mathrm{H}), 7.96(\mathrm{~s}, 1 \mathrm{H})$, $7.81 \sim 7.74(\mathrm{~m}, 2 \mathrm{H}), 7.42(\mathrm{~d}, J=8.1 \mathrm{~Hz}, 1 \mathrm{H}), 7.38(\mathrm{~d}, J=$ $8.1 \mathrm{~Hz}, 1 \mathrm{H}), 7.31 \sim 7.20(\mathrm{~m}, 4 \mathrm{H}), 6.46(\mathrm{dd}, J=17.0,10.1$ $\mathrm{Hz}, 1 \mathrm{H}), 6.27$ (dd, $J=17.0,2.1 \mathrm{~Hz}, 1 \mathrm{H}), 5.76(\mathrm{dd}, J=10.1$, $2.1 \mathrm{~Hz}, 1 \mathrm{H}), 4.90(\mathrm{~s}, 2 \mathrm{H}), 4.00(\mathrm{~s}, 3 \mathrm{H}) ;{ }^{13} \mathrm{C} \mathrm{NMR}(101$ MHz, DMSO- $\left.d_{6}\right) \delta: 165.9,163.1(\mathrm{~d}, J=363.4 \mathrm{~Hz}), 157.1$, $156.5,154.4,153.2,147.4,147.3,139.4,138.6,135.6$, $131.8,129.0,126.8,124.5(\mathrm{~d}, J=8.1 \mathrm{~Hz}), 115.1(\mathrm{~d}, J=$ $22.2 \mathrm{~Hz}), 114.7,110.6,108.5,107.6,104.2,68.5,55.9$. HRMS (ESI) calcd for $\mathrm{C}_{26} \mathrm{H}_{23} \mathrm{FN}_{5} \mathrm{O}_{4}[\mathrm{M}+\mathrm{H}]^{+} 488.1734$, found 488.1733 .

$N$-(3-(2-((4-((4-Chlorophenyl)amino)-7-methoxyquinazolin-6-yl)oxy)acetamido)phenyl)acrylamide (13j): White solid, yield $74.9 \%$. m.p. 259 $260{ }^{\circ} \mathrm{C}$; ${ }^{1} \mathrm{H}$ NMR $\left(400 \mathrm{MHz}, \mathrm{DMSO}-d_{6}\right) \delta: 10.69(\mathrm{~s}, 1 \mathrm{H}), 10.51(\mathrm{~s}, 1 \mathrm{H})$, $10.22(\mathrm{~s}, 1 \mathrm{H}), 8.52(\mathrm{~s}, 1 \mathrm{H}), 8.25(\mathrm{~s}, 1 \mathrm{H}), 8.17(\mathrm{~d}, J=8.6 \mathrm{~Hz}$, 2H), 7.47 (d, $J=8.5 \mathrm{~Hz}, 2 \mathrm{H}), 7.37$ (t, $J=7.9 \mathrm{~Hz}, 2 \mathrm{H}), 7.32$ $(\mathrm{d}, J=7.8 \mathrm{~Hz}, 1 \mathrm{H}), 6.85(\mathrm{~d}, J=2.3 \mathrm{~Hz}, 1 \mathrm{H}), 6.57(\mathrm{~d}, J=$ $2.3 \mathrm{~Hz}, 1 \mathrm{H}), 6.48(\mathrm{dd}, J=17.0,10.1 \mathrm{~Hz}, 1 \mathrm{H}), 6.30(\mathrm{dd}, J=$ 17.0, 2.2 Hz, 1H), 5.79 (dd, $J=10.1,2.2 \mathrm{~Hz}, 1 \mathrm{H}), 5.07$ (s,
2H), 3.91 (s, 3H); ${ }^{13} \mathrm{C}$ NMR (101 MHz, DMSO-d $) \delta$ : $165.7,163.2,162.7,156.7,155.1,155.0,153.5,139.5$, $138.5,138.3,131.9,129.2128 .4,126.8,126.8,123.0$, $114.8,110.5,101.3,100.7,99.4,67.9,55.7$. HRMS (ESI) calcd for $\mathrm{C}_{26} \mathrm{H}_{23} \mathrm{ClN}_{5} \mathrm{O}_{4}[\mathrm{M}+\mathrm{H}]^{+}$504.1439, found 504.1440 .

$\mathrm{N}$-(3-(2-((4-((4-Bromophenyl)amino)-7-methoxyquinazolin-6-yl)oxy)acetamido)phenyl)acrylamide (13k) White solid, yield $71.9 \%$. m.p. $178 \sim 179{ }^{\circ} \mathrm{C} ;{ }^{1} \mathrm{H}$ NMR $(400$ $\left.\mathrm{MHz}, \mathrm{DMSO}-d_{6}\right) \delta: 10.19(\mathrm{~s}, 1 \mathrm{H}), 10.13(\mathrm{~s}, 1 \mathrm{H}), 9.53(\mathrm{~s}$, $1 \mathrm{H}), 8.51(\mathrm{~s}, 1 \mathrm{H}), 8.10(\mathrm{~s}, 1 \mathrm{H}), 7.96(\mathrm{~s}, 1 \mathrm{H}), 7.81(\mathrm{~d}, J=$ $8.8 \mathrm{~Hz}, 2 \mathrm{H}), 7.57$ (d, $J=8.7 \mathrm{~Hz}, 2 \mathrm{H}), 7.39$ (dd, $J=15.4$, $8.1 \mathrm{~Hz}, 2 \mathrm{H}), 7.27(\mathrm{~s}, 2 \mathrm{H}), 6.45(\mathrm{dd}, J=17.0,10.1 \mathrm{~Hz}, 1 \mathrm{H})$, $6.26(\mathrm{dd}, J=16.9,2.2 \mathrm{~Hz}, 1 \mathrm{H}), 5.75$ (dd, $J=9.8,2.2 \mathrm{~Hz}$, $1 \mathrm{H}), 4.90(\mathrm{~s}, 2 \mathrm{H}), 4.00(\mathrm{~s}, 3 \mathrm{H}) ;{ }^{13} \mathrm{C} \mathrm{NMR}(101 \mathrm{MHz}$, DMSO- $\left.d_{6}\right) \delta$ : $165.8,163.1,156.2,154.5,153.0,147.5$, $147.4,139.4,138.8,138.6,131.8,131.2,129.1,126.9$, 124.0, 114.9, 114.8, 110.6, 108.7, 107.6, 104.2, 68.5, 56.0. HRMS (ESI) calcd for $\mathrm{C}_{26} \mathrm{H}_{23} \mathrm{BrN}_{5} \mathrm{O}_{4}[\mathrm{M}+\mathrm{H}]^{+}$548.0933, found 548.0933 .

$N$-(3-(2-((7-Methoxy-4-( $p$-tolylamino)quinazolin-6yl)oxy)acetamido)phenyl)acrylamide (131): White solid, yield 75.3\%. m.p. 157 $158{ }^{\circ} \mathrm{C}$; ${ }^{1} \mathrm{H}$ NMR (400 MHz, DMSO- $\left.d_{6}\right) \delta$ : $10.19(\mathrm{~s}, 1 \mathrm{H}), 10.12(\mathrm{~s}, 1 \mathrm{H}), 9.40(\mathrm{~s}, 1 \mathrm{H})$, $8.45(\mathrm{~s}, 1 \mathrm{H}), 8.11(\mathrm{~s}, 1 \mathrm{H}), 7.97(\mathrm{~s}, 1 \mathrm{H}), 7.64(\mathrm{~d}, J=8.1 \mathrm{~Hz}$, 2H), 7.42 (d, $J=8.1 \mathrm{~Hz}, 1 \mathrm{H}), 7.37$ (d, $J=8.1 \mathrm{~Hz}, 1 \mathrm{H})$, $7.30 \sim 7.23(\mathrm{~m}, 2 \mathrm{H}), 7.19(\mathrm{~d}, J=8.1 \mathrm{~Hz}, 2 \mathrm{H}), 6.46(\mathrm{dd}, J=$ 17.0, 10.1 Hz, 1H), 6.27 (dd, $J=17.0,2.1 \mathrm{~Hz}, 1 \mathrm{H}), 5.76$ (dd, $J=10.0,2.1 \mathrm{~Hz}, 1 \mathrm{H}), 4.89$ (s, 2H), 3.99 (s, 3H), 2.31 $(\mathrm{s}, 3 \mathrm{H}) ;{ }^{13} \mathrm{C}$ NMR $\left(101 \mathrm{MHz}, \mathrm{DMSO}-d_{6}\right) \delta: 165.9,163.1$, $156.5,154.2,153.3,147.3,147.2,139.4,138.6,136.6$, $132.5,131.8,129.0,128.8,126.9,122.6,114.8,110.6$, $108.6,107.5,104.2,68.5,55.9,20.5$. HRMS (ESI) calcd for $\mathrm{C}_{27} \mathrm{H}_{26} \mathrm{~N}_{5} \mathrm{O}_{4}[\mathrm{M}+\mathrm{H}]^{+}$484.1985, found 484.1986.

$\mathrm{N}$-(3-(2-((7-Methoxy-4-((4-methoxyphenyl)amino)quinazolin-6-yl)oxy)acetamido)phenyl)acrylamide $\quad(\mathbf{1 3 m})$ : White solid, yield 70.8\%. m.p. $150 \sim 151{ }^{\circ} \mathrm{C} ;{ }^{1} \mathrm{H}$ NMR $(400$ $\left.\mathrm{MHz}, \mathrm{DMSO}-d_{6}\right) \delta: 10.19(\mathrm{~s}, 1 \mathrm{H}), 10.11(\mathrm{~s}, 1 \mathrm{H}), 9.39(\mathrm{~s}$, $1 \mathrm{H}), 8.41(\mathrm{~s}, 1 \mathrm{H}), 8.11(\mathrm{~s}, 1 \mathrm{H}), 7.95(\mathrm{~s}, 1 \mathrm{H}), 7.61(\mathrm{~d}, J=8.5$ $\mathrm{Hz}, 2 \mathrm{H}), 7.42$ (d, $J=8.1 \mathrm{~Hz}, 1 \mathrm{H}), 7.37$ (d, $J=8.2 \mathrm{~Hz}, 1 \mathrm{H})$, $7.29(\mathrm{~d}, J=8.1 \mathrm{~Hz}, 1 \mathrm{H}), 7.23(\mathrm{~s}, 1 \mathrm{H}), 6.97$ (d, $J=8.6 \mathrm{~Hz}$, $2 \mathrm{H}), 6.46(\mathrm{dd}, J=17.0,10.1 \mathrm{~Hz}, 1 \mathrm{H}), 6.27$ (dd, $J=17.0,2.2$ $\mathrm{Hz}, 1 \mathrm{H}), 5.75$ (dd, $J=10.1,2.0 \mathrm{~Hz}, 1 \mathrm{H}), 4.89$ (s, 2H), 3.99 (s, 3H), $3.77(\mathrm{~d}, J=2.2 \mathrm{~Hz}, 3 \mathrm{H}) ;{ }^{13} \mathrm{C} \mathrm{NMR}(101 \mathrm{MHz}$, DMSO- $\left.d_{6}\right) \delta$ : $165.9,163.1,156.7,155.7,154.2,153.38$, $147.2,147.1,139.4,138.6,132.0,131.8,129.0,126.9$, 124.5, 114.8, 113.6, 110.6, 108.5, 107.5, 104.2, 68.4, 55.9, 55.2. HRMS (ESI) calcd for $\mathrm{C}_{27} \mathrm{H}_{26} \mathrm{~N}_{5} \mathrm{O}_{5}[\mathrm{M}+\mathrm{H}]$ 500.1934 , found 500.1933 .

$N$-(3-(2-((4-((2,4-Dichlorophenyl)amino)-7-methoxyquinazolin-6-yl)oxy)acetamido)phenyl)acrylamide (13n): white solid, yield 73.7\%. m.p. 163 164 ${ }^{\circ} \mathrm{C} ;{ }^{1} \mathrm{H}$ NMR $(400$ $\left.\mathrm{MHz}, \mathrm{DMSO}-d_{6}\right) \delta: 10.17(\mathrm{~s}, 1 \mathrm{H}), 10.14(\mathrm{~s}, 1 \mathrm{H}), 9.53$ (s, $1 \mathrm{H}), 8.35(\mathrm{~s}, 1 \mathrm{H}), 8.08(\mathrm{~s}, 1 \mathrm{H}), 7.91(\mathrm{~s}, 1 \mathrm{H}), 7.74(\mathrm{~d}, J=2.4$ $\mathrm{Hz}, 1 \mathrm{H}), 7.61$ (d, $J=8.6 \mathrm{~Hz}, 1 \mathrm{H}), 7.49$ (dd, $J=8.5,2.4 \mathrm{~Hz}$, $1 \mathrm{H}), 7.41(\mathrm{~d}, J=8.2 \mathrm{~Hz}, 1 \mathrm{H}), 7.35$ (d, $J=8.3 \mathrm{~Hz}, 1 \mathrm{H}), 7.26$ (q, $J=6.6,5.0 \mathrm{~Hz}, 2 \mathrm{H}), 6.45(\mathrm{dd}, J=17.0,10.1 \mathrm{~Hz}, 1 \mathrm{H})$, 
$6.25(\mathrm{dd}, J=17.0,2.2 \mathrm{~Hz}, 1 \mathrm{H}), 5.75(\mathrm{dd}, J=10.2,2.2 \mathrm{~Hz}$, $1 \mathrm{H}), 4.88(\mathrm{~s}, 2 \mathrm{H}), 3.99(\mathrm{~s}, 3 \mathrm{H}) ;{ }^{13} \mathrm{C}$ NMR (101 MHz, DMSO- $\left.d_{6}\right) \delta: 165.8,163.1,157.3,154.47,153.2,147.5$, $147.3,139.3,138.7,135.3,131.8,131.7,130.7,129.1$, $129.0,127.7,126.9,114.7,114.6,110.5,108.3,107.4$, 103.8, 68.1, 56.0. HRMS (ESI) calcd for $\mathrm{C}_{26} \mathrm{H}_{22} \mathrm{Cl}_{2} \mathrm{~N}_{5} \mathrm{O}_{4}$ $[\mathrm{M}+\mathrm{H}]^{+}$538.1049, found 538.1049.

$N$-(3-(2-((4-((3,4-Dichlorophenyl)amino)-7-methoxyquinazolin-6-yl)oxy)acetamido)phenyl)acrylamide (130): White solid, yield 75.4\%. m.p. $275 \sim 276{ }^{\circ} \mathrm{C} ;{ }^{1} \mathrm{H}$ NMR (400 MHz, DMSO- $\left.d_{6}\right) \delta$ : $10.19(\mathrm{~s}, 1 \mathrm{H}), 10.12(\mathrm{~s}, 1 \mathrm{H}), 9.60$ (s, $1 \mathrm{H}), 8.58(\mathrm{~d}, J=2.3 \mathrm{~Hz}, 1 \mathrm{H}), 8.25(\mathrm{~d}, J=2.5 \mathrm{~Hz}, 1 \mathrm{H}), 8.12$ (s, 1H), $7.94(\mathrm{~s}, 1 \mathrm{H}), 7.86(\mathrm{dd}, J=8.9,2.5 \mathrm{~Hz}, 1 \mathrm{H}), 7.63(\mathrm{~d}$, $J=8.9 \mathrm{~Hz}, 1 \mathrm{H}), 7.43(\mathrm{~d}, J=8.0 \mathrm{~Hz}, 1 \mathrm{H}), 7.38$ (d, $J=8.1$ $\mathrm{Hz}, 1 \mathrm{H}), 7.29$ (s, 2H), 6.47 (dd, $J=17.0,10.1 \mathrm{~Hz}, 1 \mathrm{H}), 6.27$ (dd, $J=17.1,2.2 \mathrm{~Hz}, 1 \mathrm{H}), 5.76(\mathrm{dd}, J=10.1,2.2 \mathrm{~Hz}, 1 \mathrm{H})$, $4.91(\mathrm{~s}, 2 \mathrm{H}), 4.01(\mathrm{~s}, 3 \mathrm{H}) ;{ }^{13} \mathrm{C}$ NMR $\left(101 \mathrm{MHz}, \mathrm{DMSO}-d_{6}\right)$ $\delta: 165.8,163.1,155.9,154.6,152.9,147.6,147.5,139.7$, $139.4,138.6,131.8,130.6,130.2,129.0,126.8,124.4$, 122.7, 121.6, 114.8, 110.6, 108.7, 107.6, 104.1, 68.6, 56.0. HRMS (ESI) calcd for $\mathrm{C}_{26} \mathrm{H}_{22} \mathrm{Cl}_{2} \mathrm{~N}_{5} \mathrm{O}_{4}[\mathrm{M}+\mathrm{H}]^{+} 538.1049$, found 538.1048 .

$\mathrm{N}$-(3-(2-((4-((3-Chloro-4-fluorophenyl)amino)-7-methoxyquinazolin-6-yl)oxy)acetamido)phenyl)acrylami (13p): White solid, yield 73.6\%. m.p. $257 \sim 258{ }^{\circ} \mathrm{C} ;{ }^{1} \mathrm{H}$ NMR $(400$ MHz, DMSO-d $\left.d_{6}\right) \delta: 10.18(\mathrm{~s}, 1 \mathrm{H}), 10.11(\mathrm{~s}, 1 \mathrm{H}), 9.56(\mathrm{~s}$, $1 \mathrm{H}), 8.54(\mathrm{~s}, 1 \mathrm{H}), 8.12(\mathrm{dd}, J=7.0,2.5 \mathrm{~Hz}, 2 \mathrm{H}), 7.94$ (s, $1 \mathrm{H}), 7.77$ (ddd, $J=9.2,4.4,2.5 \mathrm{~Hz}, 1 \mathrm{H}), 7.44(\mathrm{t}, J=9.3 \mathrm{~Hz}$, 2H), $7.38(\mathrm{~d}, J=8.1 \mathrm{~Hz}, 1 \mathrm{H}), 7.31 \sim 7.25(\mathrm{~m}, 2 \mathrm{H}), 6.46(\mathrm{dd}$, $J=17.0,10.1 \mathrm{~Hz}, 1 \mathrm{H}), 6.27(\mathrm{dd}, J=16.9,2.1 \mathrm{~Hz}, 1 \mathrm{H}), 5.76$ $(\mathrm{dd}, J=10.1,2.1 \mathrm{~Hz}, 1 \mathrm{H}), 4.90(\mathrm{~s}, 2 \mathrm{H}), 4.01(\mathrm{~s}, 3 \mathrm{H}) ;{ }^{13} \mathrm{C}$ NMR (101 MHz, DMSO- $\left.d_{6}\right) \delta: 165.8(\mathrm{~d}, J=271.7 \mathrm{~Hz})$, $156.1,154.5,153.0,152.0,147.5,147.4,139.4,138.6$, $136.7,131.8,129.0,126.8,123.5,122.3,118.9,116.6$ (d, $J$ $=21.2 \mathrm{~Hz}$ ), 114.8, 114.7, 110.6, 108.6, 107.6, 104.1, 68.6, 56.0. HRMS (ESI) calcd for $\mathrm{C}_{26} \mathrm{H}_{22} \mathrm{ClFN}_{5} \mathrm{O}_{4}[\mathrm{M}+\mathrm{H}]^{+}$ 522.1344 , found 522.1342 .

$\mathrm{N}$-(3-(2-((7-Methoxy-4-((3,4,5-trimethoxyphenyl)amino)quinazolin-6-yl)oxy)acetamido)phenyl)acrylamide (13q): White solid, yield $77.9 \%$. m.p. $234 \sim 235{ }^{\circ} \mathrm{C} ;{ }^{1} \mathrm{H}$ NMR (400 MHz, DMSO- $\left.d_{6}\right) \delta: 10.18(\mathrm{~s}, 1 \mathrm{H}), 10.11(\mathrm{~s}, 1 \mathrm{H})$, $9.39(\mathrm{~s}, 1 \mathrm{H}), 8.49(\mathrm{~s}, 1 \mathrm{H}), 8.11(\mathrm{~s}, 1 \mathrm{H}), 7.96(\mathrm{~s}, 1 \mathrm{H}), 7.42$ $(\mathrm{d}, J=8.2 \mathrm{~Hz}, 1 \mathrm{H}), 7.37(\mathrm{~d}, J=8.1 \mathrm{~Hz}, 1 \mathrm{H}), 7.29$ (d, $J=$ $8.1 \mathrm{~Hz}, 1 \mathrm{H}), 7.26$ (s, 1H), 7.17 (s, 2H), 6.45 (dd, $J=17.0$, $10.1 \mathrm{~Hz}, 1 \mathrm{H}), 6.26(\mathrm{dd}, J=17.1,2.0 \mathrm{~Hz}, 1 \mathrm{H}), 5.75(\mathrm{dd}, J=$ 10.0, $2.0 \mathrm{~Hz}, 1 \mathrm{H}), 4.91(\mathrm{~s}, 2 \mathrm{H}), 4.00(\mathrm{~s}, 3 \mathrm{H}), 3.80$ (s, 6H), $3.67(\mathrm{~s}, 3 \mathrm{H}) ;{ }^{13} \mathrm{C}$ NMR (101 MHz, DMSO- $\left.d_{6}\right) \delta: 165.9$, $163.1,156.6,154.3,153.2,152.5,147.3,147.2,139.4$, $138.6,135.3,133.8,131.8,129.0,126.9,114.8,110.6$, $108.7,107.6,104.4,100.5,68.6,60.1,55.9,55.9$. HRMS (ESI) calcd for $\mathrm{C}_{29} \mathrm{H}_{30} \mathrm{~N}_{5} \mathrm{O}_{7}[\mathrm{M}+\mathrm{H}]^{+}$560.2145, found 560.2145 .

\subsubsection{Cell culture and treatment}

Human cancer cells MCF-7, MGC-803 and PC-3, HGC27 was purchased from the China Center for Type Culture Collection (CCTCC, China) and maintained in RPMI-1640
(Solarbio, China) and dulbecco's modified eagle medium (DMEM) (Solarbio) complete medium (which supplemented with $10 \%$ fetal calf serum (FBS) and $100 \mathrm{U} / \mathrm{mL}$ penicillin and $100 \mathrm{~g} / \mathrm{mL}$ streptomycin antibiotics) at $37{ }^{\circ} \mathrm{C}$ in a humidified atmosphere of $5 \% \mathrm{CO}_{2}$.

\subsubsection{MTT assay}

Cells in the logarithmic growth phase were seeded in 96-well plates at 3000 5000 cells per well. After the cells were cultured for $24 \mathrm{~h}$, different concentrations of compounds $13 \mathbf{a} \sim \mathbf{1 3 q}$ were treated for $72 \mathrm{~h}$, respectively. MTT (3-[4,5-dimethylthiazol-2-yl]-2,5-diphenyltetrazolium bromide, Solarbio) was added to each well at a final concentration of $0.5 \mathrm{mg} / \mathrm{mL}$. After $4 \mathrm{~h}$ in a $37{ }^{\circ} \mathrm{C}$ incubator, the medium was aspirated. $150 \mu \mathrm{L}$ of dimethyl sulfoxide (DMSO) was then added to each well to dissolve the formazan, and the plate was shaken on a shaker for $10 \mathrm{~min}$. The absorbance was measured by an enzyme-linked immunosorbent assay reader (BioTek, USA) at a wavelength of $490 \mathrm{~nm}$, and the cell survival rate was measured. Viability rate $(\%)=$ Abs 490 treated cells/Abs 490 control cells $\times 100 \%$. The concentration-response curve generated by SPSS 16.0 software was used to determine the concentration of compound $\left(\mathrm{IC}_{50}\right)$ required to inhibit cell growth by $50 \%$. Cell viability curves were generated using GraphPad Prism 7.0 software at various concentrations of all compounds. Results were mean $\pm \mathrm{SD}$ of three independent experiments.

Supporting Information The ${ }^{1} \mathrm{H}$ NMR, ${ }^{13} \mathrm{C}$ NMR and HRMS of compounds $\mathbf{1 3 a} \sim \mathbf{1 3 q}$ are available for free download from our website (http://sioc-jour nal.cn/).

\section{References}

[1] Li, E. D.; Meng, Y. Q.; Zhang, L. Y.; Zhang, Y.; Wang, J. K.; Zhang, D. Q.; Song, P. P.; Xin, J. C.; Li, N.; Zheng, J. X.; Ke, Y.; Liu, H. M.; Zhang, Q. R. Chin. J. Org. Chem. 2019, 39, 2875 (in Chinese). (李二冬, 孟娅琪, 张路野, 张洋, 王继宽, 张丹青, 宋攀攀, 辛 景超，栗娜，郑甲信，可钰，刘宏民，张秋荣，有机化学，2019, 39, 2875.)

[2] Li, N.; Xin, J.; Ma, Q.; Li, E.; Meng, Y.; Bao, C.; Yang, P.; Song, P.; Cui, F.; Cheng, P.; Gu, Y.; Zhao, P.; Ke, Y.; Liu, H.; Zhang, Q. Chin. J. Org. Chem. 2018, 38, 665 (in Chinese).

(栗娜, 辛景超, 马启胜, 李二冬, 孟娅琪, 包崇男, 杨鹏, 宋攀 攀，崔飞, 陈鹏举，顾一飞, 赵培荣, 可钰, 刘宏民, 张秋荣, 有 有机化学, 2018, 38, 665.)

[3] Hua, X.; Zhang, H.; Jia, J.; Chen, S.; Sun, Y.; Zhu, X. Biomed. Pharmacother. 2020, 127, 110156 .

[4] Alagarsamy, V.; Chitra, K.; Saravanan, G.; Solomon, V. R.; Sulthana, M. T.; Narendhar, B. Eur. J. Med. Chem. 2018, 151, 628.

[5] Kabri, Y.; Azas, N.; Dumetre, A.; Hutter, S.; Laget, M.; Verhaeghe, P.; Gellis, A.; Vanelle, P. Eur. J. Med. Chem. 2010, 45, 616.

[6] Rojas Aguirre, Y.; Hernández Luis, F.; Mendoza Martínez, C.; Sotomayor, C. P.; Aguilar, L. F.; Villena, F.; Castillo, I.; Hernández, D. J.; Suwalsky, M. Biochim. Biophys. Acta 2012, 1818, 738.

[7] Ji, Q.; Yang, D.; Wang, X.; Chen, C.; Deng, Q.; Ge, Z.; Yuan, L.; Yang, X.; Liao, F. Bioorg. Med. Chem. Lett. 2014, 22, 3405.

[8] Selvam, T. P.; Sivakumar, A.; Prabhu, P. P. J. Pharm. Bioallied Sci. 2014, 6, 278 .

[9] Rakesh, K. P.; Manukumar, H. M.; Gowda, D. C. Bioorg. Med. Chem. Lett. 2015, 25, 1072.

[10] Hu, J.; Zhang, Y.; Dong, L.; Wang, Z.; Chen, L.; Liang, D.; Shi, D.; 
Shan, X.; Liang, G. Chem. Biol. Drug Des. 2015, 85, 672.

[11] Ugale, V. G.; Bari, S. B. Eur. J. Med. Chem. 2014, 80, 447.

[12] El-Azab, A. S.; Eltahir, K. E. Bioorg. Med. Chem. Lett. 2012, 22, 327.

[13] Magyar, K.; Deres, L.; Eros, K.; Bruszt, K.; Seress, L.; Hamar, J.; Hideg, K.; Balogh, A.; Gallyas, F., Jr.; Sumegi, B.; Toth, K.; Halmosi, R. Biochim. Biophys. Acta 2014, 1842, 935.

[14] Malamas, M. S.; Millen, J. J. Med. Chem. 1991, 34, 1492.

[15] Galvez, J.; Polo, S.; Insuasty, B.; Gutierrez, M.; Caceres, D.; Alzate-Morales, J. H.; De-la-Torre, P.; Quiroga, J. Comput. Biol. Chem. 2018, 74, 218.

[16] Li, E. D.; Lin, Q.; Meng, Y. Q.; Zhang, L. Y.; Song, P. P.; Li, N.; Xin, J. C.; Yang, P.; Bao, C. N.; Zhang, D. Q.; Zhang, Y.; Wang, J. K.; Zhang, Q. R.; Liu, H. M. Eur. J. Med. Chem. 2019, 172, 36.

[17] Mehndiratta, S.; Sapra, S.; Singh, G.; Singh, M.; Nepali, K. Recent
Pat. Anti-Cancer Drug Discovery 2016, 11,2

[18] Ravez, S.; Castillo-Aguilera, O.; Depreux, P.; Goossens, L. Expert. Opin. Ther. Pat. 2015, 25, 789 .

[19] Hei, Y. Y.; Shen, Y.; Wang, J.; Zhang, H.; Zhao, H. Y.; Xin, M.; Cao, Y. X.; Li, Y.; Zhang, S. Q. Bioorg. Med. Chem. 2018, 26, 2173.

[20] Patel, H.; Pawara, R.; Ansari, A.; Surana, S. Eur. J. Med. Chem. 2017, 142, 32.

[21] Shi, H.; Lai, B.; Chen, S.; Zhou, X.; Nie, J.; Ma, J.-A. Chin. J. Chem. 2017, 35, 1693.

[22] Peng, F. W.; Xuan, J.; Wu, T. T.; Xue, J. Y.; Ren, Z. W.; Liu, D. K.; Wang, X. Q.; Chen, X. H.; Zhang, J. W.; Xu, Y. G.; Shi, L. Eur. J. Med. Chem. 2016, 109, 1.

[23] Ju, Y.; Wu, J.; Yuan, X.; Zhao, L.; Zhang, G.; Li, C.; Qiao, R. J. Med. Chem. 2018, 61 .

(Lu, Y.) 